\title{
Simultaneous two-photon activation and imaging of neural activity based on spectral-temporal modulation of supercontinuum light
}

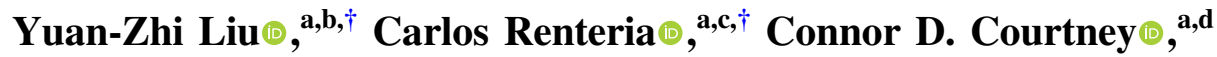 \\ Baher Ibrahim, ${ }^{\text {a }}$ Sixian You, ${ }^{\text {a,b,e }}$ Eric J. Chaney, ${ }^{\text {a }}$ Ronit Barkalifa, ${ }^{\text {a }}$ \\ Rishyashring R. Iyer, ${ }^{\text {a,b }}$ Mantas Zurauskas $\odot,{ }^{a}$ Haohua Tu, ${ }^{a}$ \\ Daniel A. Llano, a,d,f Catherine A. Christian-Hinman ${ }^{\text {a,d,f }}$

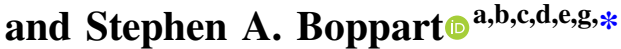 \\ ${ }^{a}$ University of Illinois at Urbana-Champaign, Beckman Institute for Advanced Science and \\ Technology, Urbana, Illinois, United States \\ ${ }^{b}$ University of Illinois at Urbana-Champaign, Department of Electrical and \\ Computer Engineering, Urbana, Illinois, United States \\ ${ }^{\mathrm{c}}$ University of Illinois at Urbana-Champaign, Department of Bioengineering, \\ Urbana, Illinois, United States \\ ${ }^{\mathrm{d}}$ University of Illinois at Urbana-Champaign, Neuroscience Program, \\ Urbana, Illinois, United States \\ eUniversity of Illinois at Urbana-Champaign, Computational Science and Engineering, \\ Urbana, Illinois, United States \\ ${ }^{f}$ University of Illinois at Urbana-Champaign, Department of Molecular and \\ Integrative Physiology, Urbana, Illinois, United States \\ ${ }^{\mathrm{g}}$ University of Illinois at Urbana-Champaign, Carle Illinois College of Medicine, \\ Urbana, Illinois, United States
}

\begin{abstract}
Significance: Recent advances in nonlinear optics in neuroscience have focused on using two ultrafast lasers for activity imaging and optogenetic stimulation. Broadband femtosecond light sources can obviate the need for multiple lasers by spectral separation for chromatically targeted excitation.

Aim: We present a photonic crystal fiber (PCF)-based supercontinuum source for spectrally resolved two-photon (2P) imaging and excitation of GCaMP6s and C1V1-mCherry, respectively. Approach: A PCF is pumped using a 20-MHz repetition rate femtosecond laser to generate a supercontinuum of light, which is spectrally separated, compressed, and recombined to image GCaMP6s (930 nm excitation) and stimulate the optogenetic protein, C1V1-mCherry (1060 nm excitation). Galvanometric spiral scanning is employed on a single-cell level for multiphoton excitation and high-speed resonant scanning is employed for imaging of calcium activity.

Results: Continuous wave lasers were used to verify functionality of optogenetic activation followed by directed $2 \mathrm{P}$ excitation. Results from these experiments demonstrate the utility of a supercontinuum light source for simultaneous, single-cell excitation and calcium imaging. Conclusions: A PCF-based supercontinuum light source was employed for simultaneous imaging and excitation of calcium dynamics in brain tissue. Pumped PCFs can serve as powerful light sources for imaging and activation of neural activity, and overcome the limited spectra and space associated with multilaser approaches. (C) The Authors. Published by SPIE under a Creative Commons Attribution 4.0 Unported License. Distribution or reproduction of this work in whole or in part requires full attribution of the original publication, including its DOI. [DOI: 10.1117/1.NPh.7.4.045007]
\end{abstract}

Keywords: two-photon optogenetics; two-photon fluorescence microscopy; calcium imaging; neurons; supercontinuum; photonic crystal fiber.

Paper 20056R received Jul. 23, 2020; accepted for publication Oct. 14, 2020; published online Nov. 3, 2020.

\footnotetext{
*Address all correspondence to Stephen A. Boppart boppart@illinois.edu

These authors have contributed equally to this work.
} 


\section{Introduction}

The use of multiple optical tools for imaging and excitation of neurons and neural tissue has grown dramatically in recent years. Researchers have successfully engineered two-photon (2P) systems for bidirectional excitation and measurement of neural activity with sophisticated optical designs. ${ }^{1-7}$ Typically, two spectrally distinct laser sources are required to reliably and independently excite fluorophores and optogenetic probes with minimal crosstalk. With a large palette of optogenetic proteins, calcium indicators, voltage indicators, and other custom-tailored indicators to monitor and induce neural activity ${ }^{8-13}$ comes the added need of multiple laser sources for bidirectional probing of neural tissue. This becomes even more striking with the ubiquitous use of ultrafast laser sources, which are needed to induce $2 \mathrm{P}$ absorption events, and thus elicit responses from individual neurons and brain regions due to the reduced out-of-focus excitation. ${ }^{3,14-19}$ Indeed, the reduced out-of-focus excitation, which can be exploited with high spatiotemporal resolution for single-cell interrogation of neural circuits, provides an added level of control for brain research that is otherwise not possible with continuous wave $(\mathrm{CW})$ illumination. This allows for the single-cell precision needed for directed, optogenetic excitation of neural circuits. These sources, however, add to the increased cost, space, and complexity of these systems, and consequently the technical expertise necessary to operate and maintain these systems. For these systems to become more widely used in the neuroscience community, more versatile systems are required. Furthermore, the broad spectrum of fluorophores of interest to neuroscientists requires spectral tuning to realize the versatility that comes with this diversity.

Advances in supercontinuum generation provide such promise. Multiphoton processes typically require ultrafast light sources to achieve the high-peak power needed to generate responses with longer-wavelength sources. Ultrafast sources have become increasingly adopted for optical imaging $^{20-24}$ to track inherent metabolic and structural properties of tissue for applications in oncology, ${ }^{23-28}$ biophysics, ${ }^{29-33}$ and neuroimaging. ${ }^{4,12,18,34-37}$ Notably, the broad spectrum of activity indicators and optogenetic proteins in neural studies, although convenient and versatile, typically require multiple laser sources. These setups are highly specific to the spectral constraints imposed by the light source. Multiple setups or dramatic changes, and thus extensive system realignment, to existing setups are required, especially for multiphoton setups. This becomes even more difficult should multiple fluorophores or optogenetic proteins with distinct spectral bands be used for experiments. These limitations can be overcome by using a coherent supercontinuum optical source and spectral-temporal pulse shaping.

Coherent supercontinuum light sources can be generated by carefully designed photonic crystal fibers (PCFs) $)^{38-42}$ and multispectral amplitude and phase shaping of light can be implemented for selective excitation of photosensitive molecules. Notably, pulse shaping using a $4 \mathrm{f}$ pulse shaper has provided the ability to reliably and selectively excite fluorophores of interest, while suppressing undesired signals. ${ }^{20}$ Although fiber-based lasers are increasing in popularity for bioimaging, ${ }^{21,25,27,38,43,44}$ the advantages of supercontinuum generation and pulse shaping are otherwise not widely implemented for microscopy. Beyond spectral selectivity, amplitude, and phase shaping of fiber-based supercontinuum facilitate imaging by providing high-power, short pulses $^{45-48}$ that improve the efficiency of the nonlinear optical processes we elicit. ${ }^{45}$ These advances and advantages of supercontinuum sources, however, have not been widely recognized in neuroscience, with most groups adopting the use of multiple femtosecond lasers for their investigations. $1,2,6,7$

In this work, we developed a multiband $2 \mathrm{P}$ excitation system based on supercontinuum generation for simultaneous $2 \mathrm{P}$ calcium imaging and $2 \mathrm{P}$ optogenetic excitation of neural tissue. A femtosecond fiber laser was used to pump a PCF for supercontinuum generation, followed by pulse-shaping of dual spectral bands to generate near transform-limited pulses, as done previously in our group. ${ }^{21}$ A spatial-light-modulator-based pulse shaper was used for spectraltemporal femtosecond pulse shaping in this work, which provides a user-friendly method for simultaneous multispectral band pulse compression that otherwise would require multiple separate pulse compressors with delicate alignment of optical dispersive elements for the specific spectral bands. This pulse shaper solely requires the user to select the spectrum of interest and to operate the corresponding pulse compression with a computer. ${ }^{49}$ These two individually compressed spectral bands were guided into two optical paths, one for the excitation of the calcium 
indicator GCaMP6s in brain tissue,${ }^{50}$ and the other for the optogenetic protein C1V1-mCherry. ${ }^{51}$ The hippocampi of transgenic GCaMP6s mice were bilaterally injected with C1V1-mCherry using a commercial AAV virus for optogenetic expression of the excitatory opsin, C1V1mCherry. The brain slices were harvested after at least 3 weeks postinjection and used for experiments. Our results demonstrate positive expression of these spectrally distinct fluorophores using the supercontinuum source and actuation of single-cell activity using optogenetics. Continuouswave lasers were used to verify the widespread excitation of activity in the brain and verify the localization of excitation seen using our ultrafast PCF source. Spiral-scanning was implemented to elicit a response from individual cell bodies. ${ }^{7}$ This work shows the utility of supercontinuum generation in neuroscience as an alternative to multilaser systems.

\section{Methods}

All animal procedures were performed in accordance with the relevant guidelines and regulations under an IACUC protocol approved by the University of Illinois at Urbana-Champaign.

\subsection{Viral Injection and Brain Slice Preparations}

A C1V1-mCherry transducing viral vector [AAV-DJ-CaMKIIa-C1V1-(E122T/E162T)-TS-P2AmCherry, Stanford Neuroscience Gene Vector and Virus Core] was injected into the dentate gyrus of hippocampi in transgenic Thy1-GCaMP6s mice (Stock \# 025776, Jackson Labs) of both sexes, 1 to 3 months old (P30 to P90). Animals were euthanized up to 3 months postinjection for experiments. Animals were anesthetized with $2 \%$ to $3 \%$ oxygen-vaporized isoflurane (Clipper Distributing Company) and placed in a stereotaxic apparatus (Kopf Instruments). Carprofen (5 mg/kg, Zoetis) was administered subcutaneously prior to surgery. The viral solution was loaded into a $10-\mu \mathrm{L}$ Nanofil syringe with a $33 \mathrm{G}$ needle driven by an injection pump controller (Micro4, World Precision Instruments). A $1-\mu \mathrm{L}$ volume of the viral solution at a titer of $\sim 10^{12}$ genomes $/ \mathrm{mL}$ was injected into the hippocampal dentate gyrus region ( $1.8 \mathrm{~mm}$ posterior to bregma, $\pm 1.3 \mathrm{~mm}$ lateral from bregma, and 1.4 ventral to the cranial surface) at $0.12 \mu \mathrm{L} / \mathrm{min}$. The syringe was left stationary 3 to $5 \mathrm{~min}$ postinjection to promote diffusion of the viral vector throughout the injection site. The wound was closed with silk sutures (Perma-hand, Ethicon). Finally, 2.5\% lidocaine $+2.5 \%$ prilocaine cream (Hi-Tech Pharmacal) and Neosporin (Johnson \& Johnson) were applied topically to the injection site to promote healing and reduce inflammation.

For slice preparation, animals were anesthetized by $2 \%$ to $3 \%$ oxygen-vaporized isoflurane, followed by decapitation. The mouse brain was then isolated and quickly transferred to chilled cutting solution ( $254 \mathrm{mM}$ sucrose, $11 \mathrm{mM}$ glucose, $2.5 \mathrm{mM} \mathrm{KCl}, 1.25 \mathrm{mM} \mathrm{NaH}_{2} \mathrm{PO}_{4}, 10 \mathrm{mM}$ $\mathrm{MgSO}_{4}, 0.5 \mathrm{mM} \mathrm{CaCl}_{2}$, and $26 \mathrm{mM} \mathrm{NaHCO}_{3}$ ). A vibratome (VT1000S, Leica Biosystems) was then used to cut 300- $\mu \mathrm{m}$-thick slices. Regions near the injection site were saved for experimentation, whereas all other brain slices were discarded. Sliced tissue was incubated in artificial cerebrospinal fluid $\left(126 \mathrm{mM} \mathrm{NaCl}, 2.5 \mathrm{mM} \mathrm{KCl}, 10 \mathrm{mM}\right.$ glucose, $1.25 \mathrm{mM} \mathrm{NaH} \mathrm{PO}_{4}$, $1 \mathrm{mM} \mathrm{MgSO}_{4}, 2 \mathrm{mM} \mathrm{CaCl}_{2}$, and $26 \mathrm{mM} \mathrm{NaHCO}_{3}$ at $298 \mathrm{mOsm}$ ) at $32^{\circ} \mathrm{C}$ for $60 \mathrm{~min}$, then transferred to room temperature for at least 15 min prior to imaging. Slices were continuously perfused with $95 \%$ oxygen and $5 \% \mathrm{CO}_{2}$ at all times.

\subsection{Calcium Imaging and Single-Cell Photostimulation Experiments}

The experimental setup is illustrated in Fig. 1. A large mode area PCF (PM-LMA-15, NKT Photonics) was pumped by a 1030-nm femtosecond fiber laser (Satsuma, Amplitude Systemes) to generate the highly polarized coherent fiber supercontinuum. ${ }^{21}$ Although the pump laser can output an average power of $10 \mathrm{~W}$, we only applied $3.5 \mathrm{~W}$ for pumping, which was more than sufficient for our experiments. The repetition rate was also tuned to $20 \mathrm{MHz}$ in these studies. The broadband coherent supercontinuum was directed to a 640 pixel, 4-f pulse shaper (MIIPS Box 640, Biophotonics Solutions Inc.) for further spectral-temporal modulation. The broadband spectrum was separated into two bands ( 890 to $950 \mathrm{~nm}$ and 1010 to $1160 \mathrm{~nm}$ ) inside the pulse 


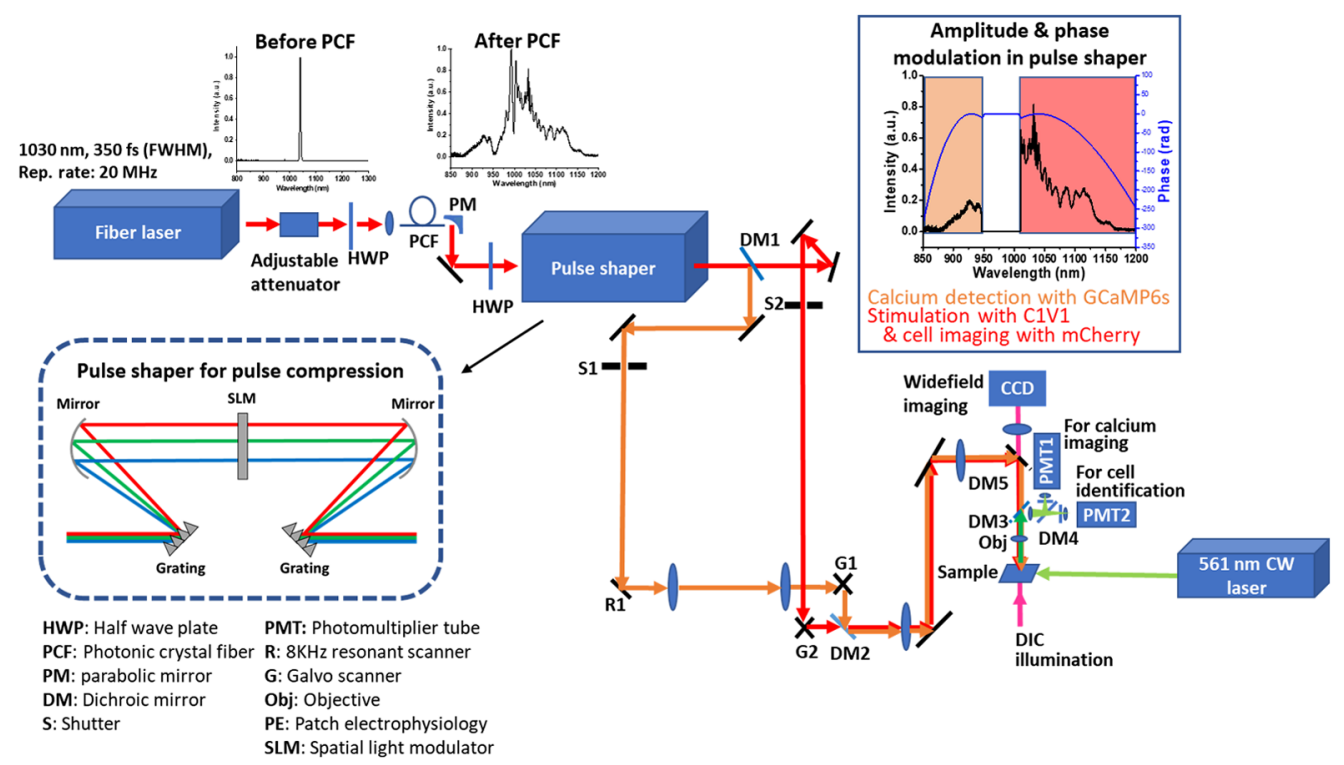

Fig. 1 Optical system used for experiments. A 1030-nm, 350-fs, and 20-MHz repetition rate laser is used to pump a PCF for supercontinuum generation. The light is directed to a pulse shaper which compresses the pulses at two bands to maximize $2 \mathrm{P}$ efficiency and improve image quality. After the pulse shaper, the light is split with a dichroic mirror to separate paths. The GCaMP6s path is driven by a resonant scanner paired to galvanometers for high-speed imaging. The C1V1mCherry path is directed to a pair of galvanometers to scan for imaging and for spiral scanning of target neurons. The two paths are recombined at the sample for experiments. Separately, a 561-nm CW laser was directed by a multimode optical fiber to the sample for widefield optogenetic activation.

shaper by a knife-edge-based amplitude modulation, which minimized the excitation crosstalk between GCaMP6s and C1V1-mCherry, as shown in the inset of Fig. 1. With the programmable capability of the spatial light modulator inside the pulse shaper, optimized band-specific phases were simultaneously applied to these two spectral bands to achieve individually near transformlimited pulse compressions ( 890 to $950 \mathrm{~nm}, \sim 51 \mathrm{fs}$ and 1010 to $1140 \mathrm{~nm}, \sim 35 \mathrm{fs}$ ). This approach of temporal modulation is more convenient and versatile compared to the common pulse compressor methods, e.g., prism/grating pairs, which require two separate sets with careful alignment, along with the need to be realigned for different spectral band applications. The relatively broad bandwidth of each spectral band, along with the low pulse repetition rate of $20 \mathrm{MHz}$, was advantageous for generating higher peak power compared to standard $80 \mathrm{MHz}$ pulses, as reported in our previous work. ${ }^{21}$ This becomes more explicit with the formal relation between peak power $P_{\text {peak }}$, average laser power $P_{\text {avg }}$, pulse duration $t_{p}$, and laser repetition rate $f_{p}$ defined as $P_{\text {peak }}=\frac{P_{\text {avg }}}{t_{p} f_{p}}$. Because higher peak power can reduce the average laser power requirement for $2 \mathrm{P}$ excitation and minimize phototoxicity and photobleaching, the low repetition rate strategy has also recently begun to be utilized within the neuroscience community. ${ }^{6,7,17}$

The beam after the pulse shaper was then split into two paths by a 980-nm long-pass filter (FF980-Di01, Semrock) (DM1) for excitation of GCaMP and C1V1-mCherry, which were recombined (DM2) after the scanners and directed (DM5) to a modified Olympus BX51 microscope. Calcium imaging was performed at video rate using an $8-\mathrm{kHz}$ resonant scanner (SC-30, Electro-Optical Products) for one transverse axis with a standard galvanometric-scanned mirror $(6215 \mathrm{H}, \mathrm{Cambridge} \mathrm{Tech})$ for the second transverse axis. For C1V1-mCherry imaging and excitation, the beam was scanned using a pair of galvanometric-mounted mirrors. Fluorescence from calcium imaging was detected during point-scanning using an analog PMT (H7422A-40, Hamamatsu) then amplified using a PMT Transimpedance amplifier (TIA60, ThorLabs). For C1V1-mCherry imaging, the fluorescence was detected using a photon counting PMT (H7421-40, Hamamatsu). All images covered a $300 \mu \mathrm{m} \times 300 \mu \mathrm{m}$ field-of-view. A movable 
Liu et al.: Simultaneous two-photon activation and imaging of neural activity...

Table 1 Stimulation protocols for Fig. 4.

\begin{tabular}{lccccc}
\hline \hline Protocol & Exposure $(\mathrm{ms})$ & Cells targeted & \# Stimulations & \# Epochs & Burst? \\
\hline CW: P1 & 500 & All & 1 & 5 & No \\
CW: P2 & 500 & All & 1 & 5 & No \\
CW: P3 & 500 & All & 1 & 5 & No \\
2P: P1 & 30 & i and ii & 20 & 1 & Yes \\
2P: P2 & 50 & v & 1 & 5 & No \\
2P: P3 & 200 & ii & 1 & 5 & No \\
\hline \hline
\end{tabular}

dichroic mirror (DM3) was placed to separate the near-infrared excitation beam from the fluorescence for PMT detection ( $735 \mathrm{~nm}$ high pass, Semrock), with an additional dichroic placed to separate the GCaMP and C1V1-mCherry fluorescence (593 nm high pass, Semrock) in front of the PMT. The DM3 mirror can be slid out for wide field fluorescence and DIC imaging with a CCD camera (QImaging Retiga Electro). Filters were implemented for detection of GCaMP6s and C1V1-mCherry fluorescence at $510 \pm 42 \mathrm{~nm}$ and $641 \pm 37.5 \mathrm{~nm}$, respectively (FF01-510/ 84-25 and F-F02-641/75-25, Semrock). The sample was illuminated with $\sim 18 \mathrm{~mW}$ of power for excitation and imaging of C1V1-mCherry. About $18 \mathrm{~mW}$ of power was used for imaging GCaMP6s in brain tissue. Experiments were also conducted with a CW laser source operating at a 561-nm wavelength (Sapphire, Coherent). A partially under-filled objective (XLPlan N 25x 1.05 NA, Olympus) was used for all experiments. Previous studies have demonstrated that a lower effective numerical aperture, rather counter-intuitively, improves $2 \mathrm{P}$ optogenetic activation in brain tissue due to the increased number of excited opsins. ${ }^{52,53}$

For optogenetic experiments, the brain tissue was first examined by widefield GCaMP imaging, followed by C1V1-mCherry imaging, to verify the presence of GCaMP6s and determine regions with dense C1V1-mCherry expression. Once a region was established, the optogenetic stimulation protocol (both $\mathrm{CW}$ and $2 \mathrm{P}$ ) was used to evoke responses from cells near transduced areas. A spiral scanning approach was implemented for 2P stimulation, with controlled exposure times, as reported in Table 1. For CW stimulation, widefield illumination across the region of interest was employed, targeting all cells in the field-of-view. Stimulation protocols were initiated after a wait time of at least $1 \mathrm{~min}$ after identifying C1V1-mCherry positive regions to promote recovery of the proteins to their resting, ground state. Similarly, cells illuminated with either $\mathrm{CW}$ or $2 \mathrm{P}$ illumination were given adequate time $(>1 \mathrm{~min})$ to recover prior to subsequent stimulation. The response was then visualized in real time and the data were recorded for postprocessing. All data were processed offline using custom-written MATLAB software. All hardware for imaging and stimulation protocols were controlled using a custom-written LabVIEW program.

\subsection{Image Denoising}

To reduce the presence of noise inherent in fluorescent microscopy systems, image denoising was implemented prior to image analysis. In particular, the Noise2Void denoising technique ${ }^{54}$ was implemented to remove sources of noise from the captured calcium imaging sequences. Calcium transients were visible without the algorithm, but the image quality was dramatically improved, which allowed for ready identification of sources of these dynamic changes. The algorithm was run in PyCharm. Representative calcium imaging datasets demonstrating the efficacy and reliability of Noise2Void are shown in Fig. S1 in the Supplementary Materials. A representative video demonstrating the effectiveness of denoising is also included in Video S1 [URL: https://doi.org/10.1117/1.NPh.7.4.045007.1]. Additionally, the signal-to-noise ratio is quantified before and after denoising in Fig. S2 in the Supplementary Materials, quantified using the equation, $\mathrm{SNR}=10 \log _{10}\left(\frac{\mu_{\mathrm{sig}}}{\mu_{\mathrm{bckg}}}\right)$, where $\mu_{\text {sig }}$ and $\mu_{\mathrm{bckg}}$ represent the mean intensity from an image representative of signal and background, respectively. 


\subsection{Data Analysis}

All analyses were performed offline using custom MATLAB software, using a previously devised algorithm. ${ }^{55}$ Calcium transients were isolated from individual cells by selecting a point on the soma and averaging around a five-pixel crosshair. Raw images were saved in binary format. Videos were processed with a mean projection along time using ImageJ to generate the final images, unless otherwise noted. Stimulation times were validated by measuring the image power throughout the time series.

\section{Results}

\subsection{Imaging and Transduction Validation}

Initial experiments were designed to ensure that the imaging parameters were optimal for both beam paths from the supercontinuum source. Brain slices from GCaMP6s mice injected with C1V1-mCherry were imaged with and without pulse compression to demonstrate image quality optimization. Results highlighted in Fig. 2 show images of these cells and consequently the validation of the expected emission for the desired spectral bands. In particular, Fig. 2(a) shows fluorescence from hippocampal tissue both before and after pulse compression, where the green channel is GCaMP6s and the red channel is mCherry. The results verify both that adequate detection of sufficient image quality can be obtained with the system and that pulse compression does indeed result in improving the signal strength via enhanced $2 \mathrm{P}$ absorption efficiency. The line plots in Fig. 2(b), which correspond to the region highlighted by the blue dashed line in Fig. 2(a), show a cross section of the image that further exemplifies the increased signal obtained by pulse compression. Histograms of the pixel intensities of these images also illustrate the dramatic increase in signal after pulse compression. Figure 2(c) clearly illustrates a larger distribution of higher intensity pixels after pulse compression for both GCaMP6s and C1V1-mCherry.

(a)

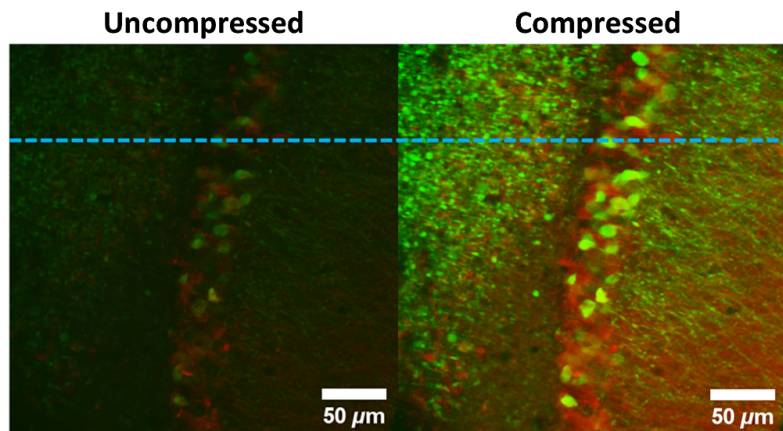

(b)
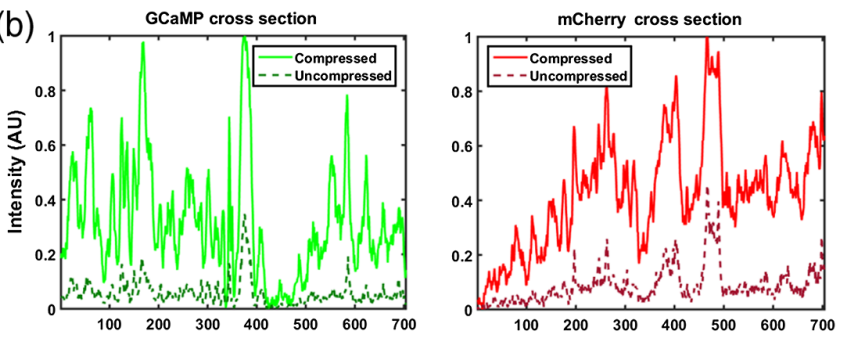

(c)
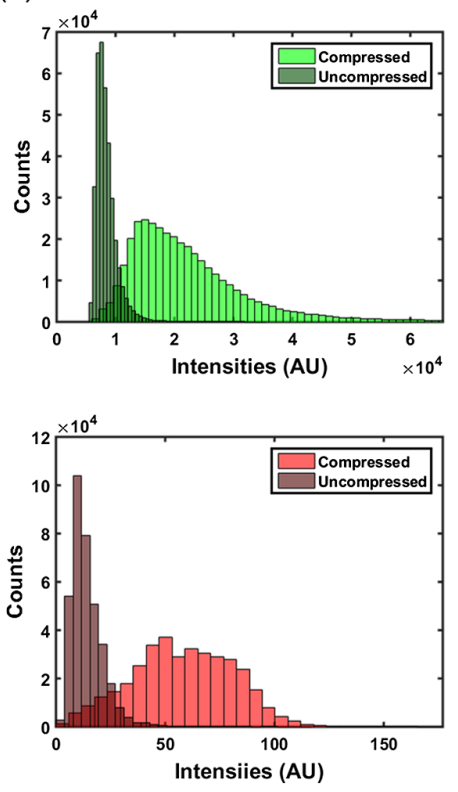

Fig. 2 Image pulse compression characterization. (a) Images of hippocampal brain slices imaged with the optical setup show a dramatic increase in image quality after pulse compression. (b) A cross section of the image (green and red) is also plotted with and without pulse compression for both the intended GCaMP6s and C1V1-mCherry channels, respectively. The cross sections were normalized to the peak intensity for the compressed images. (c) The histograms also quantify the considerable increase for both the GCaMP (top) and mCherry (bottom) images, before and after pulse compression. Notably, the distribution of intensities from the samples are much broader and illustrate a clear increase in signal intensity overall. Scale bars represent $50 \mu \mathrm{m} ; n=1$ slice, 1 mouse. 
The presence of visible structures in the sample in Fig. 2(a), along with the line plots in Fig. 2(b) and histograms in Fig. 2(c), together highlight the efficacy of the system for the desired wavelengths, and the improved image quality following pulse compression optimization.

Similarly, brain slices harvested from GCaMP6s-positive mice were imaged under the microscope to verify image quality and successful transduction of the targeted sites. Figures 3(a) and 3 (c) show widefield fluorescence images of C1V1-mCherry from the harvested brain slices using a $4 \times$ objective (Olympus). Here we see positive expression of C1V1-mCherry in neurons, which verifies the virus reached the intended delivery site. Further multiphoton imaging with the optical setup in Figs. 3(b) and 3(d) shows expression of GCaMP-positive neurons, in addition to structures of the hippocampus from C1V1-mCherry. An additional slice preparation in Fig. 3(c), coupled with the 2P imaging results from Fig. 3(d), demonstrates the reliability of the system for producing high-quality images for the fluorophores of interest. Collectively, these results demonstrate the capabilities of the system to visualize the intended fluorescent structures at targeted injection sites.

\subsection{Simultaneous Imaging and Stimulation}

Finally, verification that this system could simultaneously perform $2 \mathrm{P}$ calcium imaging and $2 \mathrm{P}$ excitation of C1V1-mCherry was performed. Expression for mCherry is shown in Figs. 3(a) and 3(c), and both GCaMP6s and C1V1-mCherry are shown in the multiphoton images in the remainder of Fig. 3, as well as in Figs. 4(a) and 4(c). Sites visibly present with GCaMP6s, C1V1-mCherry, and co-expression of the two can thus be easily identified. Expression of GCaMP6s can clearly be seen throughout the tissue and targeted delivery of C1V1-mCherry expresses well throughout the targeted region of the hippocampus. These validate, again, that targeted delivery of the virus and the genetic expression was successful. Next, actuation of the opsin was validated by excitation with a $\mathrm{CW}$ laser source. As previous literature has shown that the efficiency for absorption in opsins is significantly higher for $\mathrm{CW}$ excitation than $2 \mathrm{P}^{52}$ this

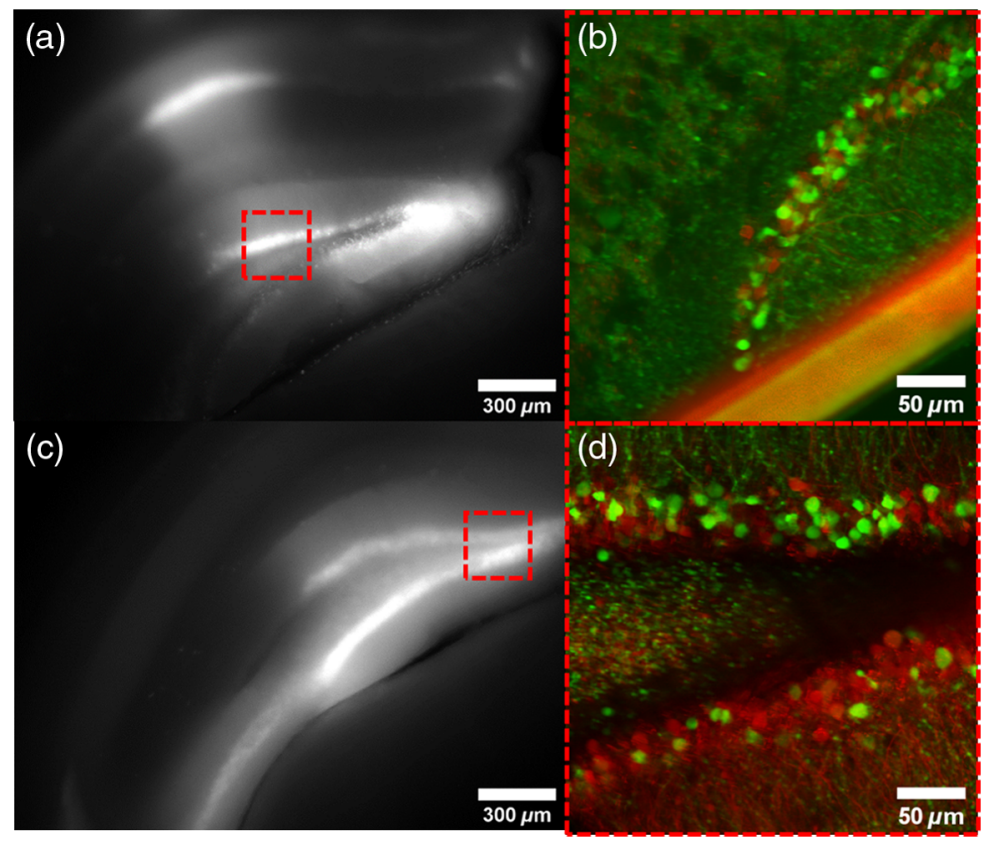

Fig. 3 Brain tissue expressing GCaMP and C1V1-mCherry. (a), (c) Widefield fluorescence imaging of two separate slice preparations shows very bright fluorescence in distinct regions of the hippocampus. (b), (d) Magnified 2P images corresponding to the areas in the red dashed boxes in the widefield images on the left illustrate positively transduced cells throughout the mouse brain at different brain sites. A wire harp was used to keep the tissue stationary, and one wire strand is shown in (b) as a large diagonal bar across the hippocampus. (a), (c) $4 \times$ scale bars represent $300 \mu \mathrm{m}$. (b), (d) $20 \times$ scale bars represent a distance of $50 \mu \mathrm{m} ; n=2$ slices, 2 mice. 
(a)

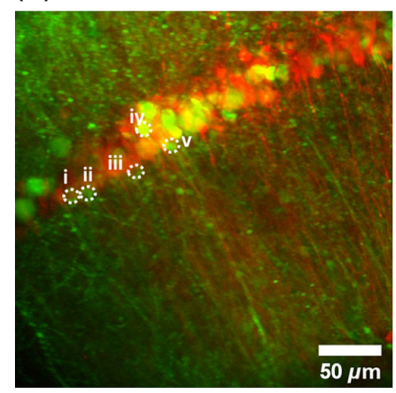

(c)

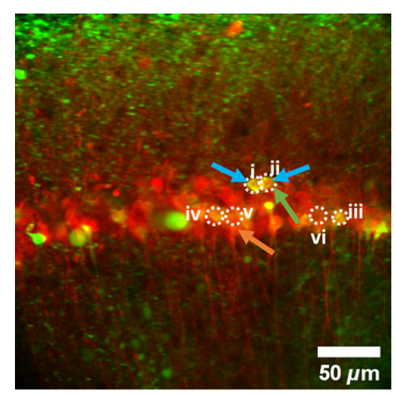

(b)

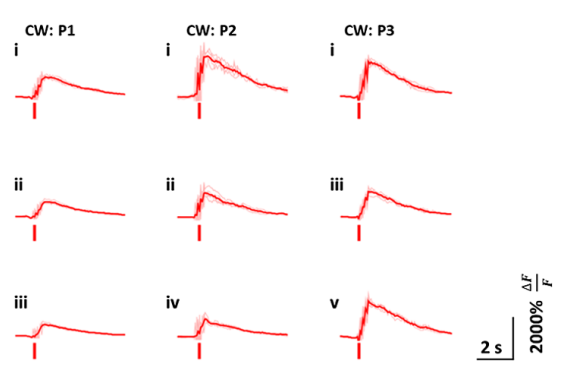

(d)

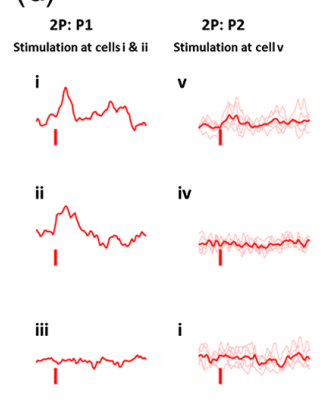

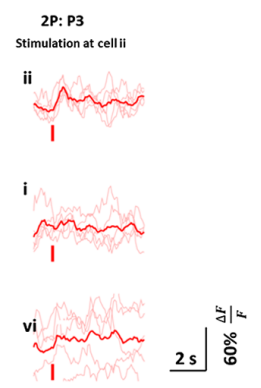

Fig. 4 Photoactivation of GCaMP using (a), (b) single-photon excitation of C1V1-mCherry and (c), (d) from multiphoton excitation. The red tick marks in (b) and (d) represent the time of the optical stimulus. Three separate stimulation protocols for CW (CW: P1 to P3) and 2P stimulation (2P: P1 to $P 3$ ) were run for each sample. For $\mathrm{CW}$ excitation, there was widefield illumination (b), whereas for $2 \mathrm{P}$ protocols (d), a single area was targeted. In (c), the blue arrows highlight the cells excited for protocol 2P: P1, the orange arrow indicates the targeted cell for protocol 2P: P2, and the green arrow indicates the cell targeted in 2P: P3. Cells were labeled between (i) and (vi), and their calcium signals are plotted for each dataset (b), (d). Red traces represent the mean signal from the calcium traces plotted in pink. Data from protocol P1 under 2P stimulation (d) had a single, burst stimulation epoch, and hence, no individual trials. CW light was incident for $500 \mathrm{~ms}$. Multiphoton sources were incident on the sample for $30 \mathrm{~ms}$ for protocol P1, $50 \mathrm{~ms}$ for protocol P2, and $200 \mathrm{~ms}$ for protocol P3. Image scale bars represent $50 \mu \mathrm{m} ; n=5$ cells under CW stimulation; and $n=6$ cells under multiphoton stimulation. Both $\mathrm{CW}$ and multiphoton protocols were performed on the same brain slice. Calcium plot scale bars represent $60 \%(2 P)$ or $2000 \%$ (single photon) $\frac{\Delta F}{F}$ on the $y$ axis, and $2 \mathrm{~s}$ on the $x$ axis.

was performed as an initial validation that the opsin was functional. Stimulation protocols for both $\mathrm{CW}$ and 2P illumination are summarized in Table 1 for ease of reference. Figure 4(b) shows the calcium response of wide-field excitation of the transduced sample with a 561-nm CW laser $\left(\sim 2 \mathrm{~mW} / \mathrm{mm}^{2}\right)$, corresponding to the locations in Fig. 4(a). Immediately, a dramatic increase in signal from GCaMP6s was evoked and captured from dozens of cells. Plots from three representative cells are shown in Fig. 4(b), with the average response (red) across multiple stimulation epochs $\left(n_{\text {epochs }}=5\right)$ superimposed on individual trials (pink). The experiment was repeated three times [Fig. 4(b), 1P: P1 to P3] to demonstrate the repeatability of these responses. These data verify the presence of each probe of interest, and the capability of both eliciting and detecting neural responses. Furthermore, they illustrate the capability of monitoring dynamic calcium transients, optogenetically evoked or otherwise.

Finally, single-cell excitation and imaging using the supercontinuum source was tested. Initial reference images of GCaMP6s and C1V1-mCherry were acquired and used to guide localization of the stimulation beam. Thereafter, a cell of interest was selected, and the stimulation protocol was initiated. Two-photon-excited imaging of GCaMP6s was performed at a $13.12-\mathrm{Hz}$ framerate, whereas $2 \mathrm{P}$ stimulation pulses with 30,50 , and $200 \mathrm{~ms}$ exposure were applied to cells i/ii, v, and ii, respectively. The results are shown in Figs. 4(c) and 4(d), where protocol 2P: P1 corresponds to the 30-ms stimulus applied to cells i and ii, 2P: P2 the 50-ms stimulus at cell v, and 2P: P3 to the 200-ms stimulus at cell ii. In our studies, protocols were 
defined by the cells targeted, the scanning geometry, and the stimulation time, as detailed in Sec. 2 and Table 1. Protocol 2P: P1 had only a single-stimulation epoch, compared to five for all other protocols. Previous literature has demonstrated that repeated $2 \mathrm{P}$ optical stimuli in a given timeframe result in more reliable calcium responses, ${ }^{7}$ and this tested for this particular protocol. For ease of reference, all cells stimulated under different protocols at the same site are identified in Fig. 4(c), with the blue arrows specifying cells stimulated under protocol 2P: P1, the orange arrow specifying the identified cell for protocol 2P: P2, and the green arrow the cell targeted under protocol 2P: P3. Spiral scanning was implemented for excitation of the entire cell to elicit sufficient $2 \mathrm{P}$ responses. Notably, the calcium transients for each protocol resulted from a single or pair of activated cell(s) near the stimulation site, rather than from several as with CW excitation. This suggests the beam was localized to a designated spot as intended for each protocol, and consequently, resulted in cells within a confined region being excited. This is especially clear for protocols 2P: P2 and 2P: P3, where cells v and ii, respectively, were targeted and showed a clear response, coupled with consistent increases in intensity for each stimulation epoch as well. In contrast, cells iv and i for protocol 2P: P2 and cells i and vi in protocol 2P: P3 did not respond. When a targeted region encompassing two neighboring cells was activated, as in Fig. 4(d) (protocol 2P: P1), there was an increase in fluorescence from both cells $i$ and ii at the time of stimulation, with an absence thereof for cell iii. This provides further support that the targeted regions alone resulted in a calcium response. Unlike the widefield $\mathrm{CW}$ illumination, $2 \mathrm{P}$ protocols targeted different single cells. These targeted regions, again, show cells at the site of interest that responded to the optical stimulus. More specifically, cells i and ii in protocol 2P: P1, cell $\mathrm{v}$ in protocol 2P: P2, and cell ii in protocol 2P: P3 all showed a clear and consistent response to $2 \mathrm{P}$ activation. In each case, the targeted cell showed, on average, an increase in fluorescence.

Notably, when cell ii was stimulated individually, in contrast to simultaneously stimulating both cells $\mathrm{i}$ and ii in protocol 2P: P1, only cell ii showed a clear response. Figure 4(d) along protocol 2P: P1 targeted both cells for stimulation, whereas protocol 2P: P3 targeted only cell ii. Also the calcium signals evoked with $\mathrm{CW}$ excitation are considerably higher than those for $2 \mathrm{P}$ activation, which is consistent with reduced amplitudes from patch-clamp recordings reported from other groups..$^{52,56}$ These results provide evidence that in addition to imaging, supercontinuum generation is useful for neural applications and optogenetics, reducing the need for complex multilaser systems, associated space occupation, and flexibility with regards to multispectral excitation.

\section{Discussion}

Presented here is a novel supercontinuum light source and system for simultaneous, multispectral excitation, detection, and imaging of neural activity. Although recent work has demonstrated supercontinuum sources for nonlinear imaging, none has shown the ability to use these sources for simultaneous neural excitation and imaging. Rather, multispectral imaging studies have used a definitive band of the supercontinuum that is optimal for excitation of multiple fluorophores and sufficient for detection of multiharmonic signals. ${ }^{21}$ This work demonstrates the utility of supercontinuum sources beyond this, using spectrally distinct beam paths from the same source to elicit excitation of spectrally distinct molecules. This is critical in simultaneous imaging and stimulation experiments to avoid undesired excitations, such as exciting opsins with the imaging beams. ${ }^{1,7,57}$ Supercontinuum sources promote flexibility for sufficient spectral separation to minimize crosstalk. The benefit of multiphoton excitation for reduced out-of-focus excitation, coupled to the flexibility of spectral separation, makes them ideal for these experiments. Moreover, the reduced cost of using a single ultrafast laser for these types of experiments reduces financial barriers associated with the multilaser approaches. The PCF used in this work is commercially available and costs $\$ 95$ for the length of fiber used. The dichroic mirror used to split the supercontinuum light is about $\$ 750$. Compared to purchasing two lasers, this is a much more economical approach. However, it should be noted that the pulse shaper used in this work is a high-end model and comparable to the cost of a separate laser. It does, however, provide the versatility for precise spatiotemporal control of ultrafast pulses and consequently a high degree of control over experimental variables. For these types of experiments, however, a high-end pulse shaper is not required. Near transform-limited pulses can easily be generated using chirped 
mirrors or prism pairs and are widely used in multiphoton microscopy for pulse compression. Use of these pulse-compression methods with a supercontinuum source, in theory, still manage to maximize beam quality while circumventing the need for a second laser source, which may still require the implementation of these compression techniques regardless. Thus the added multispectral capabilities from a single supercontinuum source can be easily and inexpensively generated.

Coherent supercontinuum sources provide additional benefits beyond spectral selectivity with regards to optogenetics. The literature has shown previously that compared to $\mathrm{CW}$ optogenetic excitation, $2 \mathrm{P}$ excitation has shown poorer efficiency for eliciting neural responses. The first experiments demonstrating $2 \mathrm{P}$ excitation of single cells theoretically and experimentally showed that $2 \mathrm{P}$ excitation, although capable of eliciting action potentials, resulted in weaker peak currents and voltages than monochromatic sources. ${ }^{52}$ This is attributable to several factors, including the excitation volume, the $2 \mathrm{P}$ absorption cross section of channelrhodopsin, exposure time, power, and various other factors. These have since been overcome through spiral scanning and spatial-temporal wavefront shaping to cover entire cell somas and temporal shaping to modify the focal volume along the optical axis ${ }^{58}$ Each of these comes with their own detriments, including reduced spectral bandwidth due to dispersion in the case of temporal focusing, ${ }^{4}$ and the dependence on reliable scanning geometries and decreased speed for scanning approaches. ${ }^{7,19}$ Still, the benefit of reliable single-cell activation and the simultaneous activation of ensembles of cells with single-cell, high temporal resolution that can be realized with $2 \mathrm{P}$ illumination, far outweighs the detriment imposed by the lower peak currents. Furthermore, use of longer-wavelength light sources promotes an increased penetration depth that is advantageous for deep tissue and in vivo imaging. Invasive surgical procedures are necessary for $\mathrm{CW}$ illumination to target a directed brain region and are still limited by increased out-of-focus excitation. Multiphoton sources, in contrast, overcome both limitations. Recent work has demonstrated that temporal phase shaping of the excitation pulse, rather than removal of spectral information, is not only sufficient for eliciting excitations, but also modifies the resulting response from the whole-cell. ${ }^{33}$ This suggests that with carefully tailored optical pulses, this $2 \mathrm{P}$ excitation efficiency can be overcome or otherwise altered, and even be used to elicit different responses on a single and multicell level.

This coherent control principle, coupled with the multispectral and compressible capabilities of supercontinuum sources, could in theory be used to optimize the excitation efficiency of a broad array of opsins. This principle has also been demonstrated in fluorescence imaging ${ }^{20}$ but much less investigated in opsins and similar photosensitive proteins. Further research will investigate the capabilities and implications of this combined approach, especially in the context of interneural communication. Still, supercontinuum sources are limited in that they require regular maintenance and skilled personnel to accompany their use. Fiber quality needs to be optimized, maintained, and replaced when damaged. Although maintenance is straightforward, training to ensure PCF output quality is required for new personnel. As this technology is furthered, efforts toward facile implementation that overcomes the need for skilled personnel will be pursued. Additionally, output power after a PCF is dependent on the coupling efficiency of the fiber and power for each spectrum on nonlinear conversion during supercontinuum generation. This, again, requires trained personnel to optimize, and a system design that must be based on the output spectrum that can be provided by the fiber. This design consideration can be limiting if insufficient power is available for a desired spectral band.

Multicell, multispectral excitation could, in theory, be used with multiple opsins to excite, inhibit, and track neural dynamics. Metabolic information can be tracked simultaneously with optogenetic excitation to track the energy demands that different excitation parameters place on neural tissue, just as it has in other settings ${ }^{23,59-62}$ and can be readily implemented using PCFs. For multicell excitation experiments, a sufficiently large amount of power is needed to provide adequate power spectral density for optogenetic activation. ${ }^{7,18}$ In our study, after splitting the beam, we find $\sim 90 \mathrm{~mW}$ can be obtained for the $920-\mathrm{nm}$ band and $\sim 500 \mathrm{~mW}$ for the $1060-\mathrm{nm}$ band. Only a small fraction of this power budget is used in the experiments here. Higher pump power also provides higher output power of supercontinuum, if necessary. Given the high image quality from the GCaMP6s channel, there is more than enough power in the imaging band to facilitate real-time imaging of calcium transients. Conveniently, the laser source used in this study has a tunable repetition rate, which further facilitates investigations of the laser parameters 
used in microscopy studies. With trends toward lower repetition rate lasers, power budget limitations can be more easily overcome to promote adequate excitation of multiple cells in a sample by the inherent increase in $2 \mathrm{P}$ absorption. By experimenting with the optical parameters, including illumination volume, scanning trajectory, repetition rate, pulse width, and power among other variables, optimal parameters can be determined to optimize $2 \mathrm{P}$ optogenetic activation. By leveraging these, more sophisticated neural circuit experiments can be realized.

\section{Conclusions and Outlook}

We present a single-source approach for imaging and optogenetic control of neural activity using supercontinuum generation. Stereotaxic injections in the hippocampi of transgenic GCaMP6s mice with C1V1-mCherry were performed to test the utility of this source for all-optical neural interrogation. The presence of C1V1-mCherry was verified in the designated injection site with $2 \mathrm{P}$ imaging and was used to guide $2 \mathrm{P}$ neural activation. $\mathrm{CW}$ excitation was performed to verify that neural tissues were expressive and responsive. Two-photon excitation was then performed while imaging GCaMP6s transients, and localized cellular responses were elicited. These results demonstrate the utility of supercontinuum and PCFs for neuroscience studies, which would benefit the neuroscience community by providing a flexible and less expensive alternative to traditional multilaser multiphoton setups.

\section{Appendix}

Video S1 compares representative calcium imaging data before (left) and after (right) denoising.

\section{Disclosures}

S. A. B and H. T. are co-founders of LiveBx, LLC, which develops laser systems for scientific applications. S. A. B., H. T., and S. Y. are inventors on patents related to supercontinuum imaging in the life sciences. All other authors declare no competing interests.

\section{Acknowledgments}

The authors would like to thank Darold Spillman for his administrative support. The authors would like to also acknowledge Adam Packer, Courtney Sobieski, Kush Paul, and Parijat Sengupta for their insightful discussions and consultations for these experiments. We would also like to thank Karl Deisseroth (Stanford University) for access to the virus. This research was supported in part by grants from the Air Force Office of Scientific Research (No. FA955017-1-0387), the National Science Foundation (No. EAGER CBET 18-41539), the National Institute of Biomedical Imaging and Bioengineering of the National Institutes of Health under Award No. T32EB019944, and the National Institutes of Health (Nos. R01 CA241618 and R43 MH119979). The content is solely the responsibility of the authors and does not necessarily represent the official views of these federal agencies. We would also like to thank the Sloan University Center of Exemplary Mentoring at Illinois for their support.

\section{Data, Code, and Material Availability}

The code used to generate the results in this manuscript is available from the corresponding author upon reasonable request and through collaborative investigations.

\section{References}

1. A. Packer et al., "Simultaneous all-optical manipulation and recording of neural circuit activity with cellular resolution in vivo," Nat. Methods 12(2), 140-146 (2015). 
2. W. Yang and R. Yuste, "In vivo imaging of neural activity," Nat. Methods 14(4), 349-359 (2017).

3. L. Carrillo-Reid et al., "Imprinting and recalling cortical ensembles," Science 353(6300), 691-694 (2016).

4. E. Papagiakoumou, E. Ronzitti, and V. Emiliani, "Scanless two-photon excitation with temporal focusing," Nat. Methods 17(6), 571-581 (2020).

5. S. Paluch-Siegler et al., "All-optical bidirectional neural interfacing using hybrid multiphoton holographic optogenetic stimulation," Neurophotonics 2(3), 031208 (2015).

6. Z. Zhang et al., "Closed-loop all-optical interrogation of neural circuits in vivo," Nat. Methods 15(12), 1037-1040 (2018).

7. W. Yang et al., "Simultaneous two-photon imaging and two-photon optogenetics of cortical circuits in three dimensions," Elife 7, e32671 (2018).

8. T. Chen et al., "Ultrasensitive fluorescent proteins for imaging neuronal activity," Nature 499(7458), 295-300 (2013).

9. V. Pérez and T. Nagai, "Genetically encoded $\mathrm{Ca}(2+)$ indicators: properties and evaluation," Biochim. Biophys. Acta 1833(7), 1787-1797 (2013).

10. C. Grienberger and A. Konnerth, "Imaging calcium in neurons," Neuron 73(5), 862-885 (2012).

11. D. Peterka, H. Takahashi, and R. Yuste, "Imaging voltage in neurons," Neuron 69(1), 9-21 (2011).

12. M. Zuend et al., "Arousal-induced cortical activity triggers lactate release from astrocytes," Nat. Metab. 2(2), 179-191 (2020).

13. R. Prakash et al., "Two-photon optogenetic toolbox for fast inhibition, excitation and bistable modulation," Nat. Methods 9(12), 1171-1179 (2012).

14. C. Xu and W. Webb, "Measurement of two-photon excitation cross sections of molecular fluorophores with data from 690 to 1050 nm," J. Opt. Soc. Am. B 13(3), 481 (1996).

15. C. Xu et al., "Multiphoton fluorescence excitation: new spectral windows for biological nonlinear microscopy," Proc. Natl. Acad. Sci. U. S. A. 93(20), 10763-10768 (1996).

16. W. Denk, J. Strickler, and W. Webb, "Two-photon laser scanning fluorescence microscopy," Science 248(4951), 73-76 (1990).

17. K. Charan et al., "Fiber-based tunable repetition rate source for deep tissue two-photon fluorescence microscopy," Biomed. Opt. Express 9(5), 2304-2311 (2018).

18. V. Emiliani et al., "All-optical interrogation of neural circuits," J. Neurosci. 35(41), 13917-13926 (2015).

19. D. Oron et al., "Two-photon optogenetics," Progress in Brain Research, 1st ed., Vol. 196, Elsevier B.V., Oxford (2012).

20. J. Ogilvie et al., "Use of coherent control for selective two-photon fluorescence microscopy in live organisms," Opt. Express 14(2), 759-766 (2006).

21. S. You et al., "Intravital imaging by simultaneous label-free autofluorescence-multiharmonic microscopy," Nat. Commun. 9(1), 2125 (2018).

22. M. Skala et al., "In vivo multiphoton microscopy of NADH and FAD redox states, fluorescence lifetimes, and cellular morphology in precancerous epithelia," Proc. Natl. Acad. Sci. U. S. A. 104(49), 19494-19499 (2007).

23. A. Shah et al., "In vivo autofluorescence imaging of tumor heterogeneity in response to treatment," Neoplasia 17(12), 862-870 (2015).

24. M. Skala et al., "In vivo multiphoton fluorescence lifetime imaging of protein-bound and free nicotinamide adenine dinucleotide in normal and precancerous epithelia," J. Biomed. Opt. 12(2), 024014 (2007).

25. S. You et al., "Slide-free virtual histochemistry (Part II): detection of field cancerization," Biomed. Opt. Express 9(11), 5253 (2018).

26. S. You et al., "Real-time intraoperative diagnosis by deep neural network driven multiphoton virtual histology," npj Precis. Oncol. 3(1), 33 (2019).

27. H. Tu et al., "Stain-free histopathology by programmable supercontinuum pulses," Nat. Photonics 10(8), 534-540 (2016).

28. B. Shen et al., "Label-free whole-colony imaging and metabolic analysis of metastatic pancreatic cancer by an autoregulating flexible optical system," Theranostics 10(4), 1849-1860 (2020). 
29. J. Ogilvie et al., "Fourier transform measurement of two-photon excitation spectra: applications to microscopy and optimal control," Opt. Lett. 30(8), 911-913 (2005).

30. V. Tiwari et al., "Spatially-resolved fluorescence-detected two-dimensional electronic spectroscopy probes varying excitonic structure in photosynthetic bacteria," Nat. Commun. 9(1), 4219 (2018).

31. J. Pan et al., "Ultrafast energy transfer within the photosystem II core complex," $P C C P$ 19(23), 15356-15367 (2017).

32. A. Loukianov et al., "Two-dimensional electronic stark spectroscopy," J. Phys. Chem. Lett. 8(3), 679-683 (2017).

33. K. Paul et al., "Coherent control of an opsin in living brain tissue," Nat. Phys. 13(11), 1111-1116 (2017).

34. Y. Zhao et al., "Two-photon microscope using a fiber-based approach for supercontinuum generation and light delivery to a small-footprint optical head," Opt. Lett. 45(4), 909-912 (2020).

35. J. Stobart et al., "Cortical circuit activity evokes rapid astrocyte calcium signals on a similar timescale to neurons," Neuron 98(4), 726-735.e4 (2018).

36. B. Li et al., "An adaptive excitation source for high-speed multiphoton microscopy," Nat. Methods 17(2), 163-166 (2020).

37. D. Ouzounov et al., "GCaMP6 $\Delta \mathrm{F} / \mathrm{F}$ dependence on the excitation wavelength in 3-photon and 2-photon microscopy of mouse brain activity," Biomed. Opt. Express 10(7), 3343-3352 (2019).

38. H. Tu and S. A. Boppart, "Coherent fiber supercontinuum for biophotonics," Laser Photonics Rev. 7(5), 628-645 (2013).

39. Y. Liu, H. Tu, and S. A. Boppart, "Wave-breaking-extended fiber supercontinuum generation for high compression ratio transform-limited pulse compression," Opt. Lett. 37(12), 2172-2174 (2012).

40. J. Hult, "A fourth-order Runge-Kutta in the interaction picture method for simulating supercontinuum generation in optical fibers," J. Lightwave Technol. 25(12), 3770-3775 (2007).

41. H. Tu et al., "Coherent fiber supercontinuum laser for nonlinear biomedical imaging," Proc. SPIE 8553, 85530A (2012).

42. H. Tu et al., "Scalar generalized nonlinear Schrödinger equation-quantified continuum generation in an all-normal dispersion photonic crystal fiber for broadband coherent optical sources," Opt. Express 18(26), 27872-27884 (2010).

43. S. A. Boppart et al., "Simultaneous label-free autofluorescence-multiharmonic microscopy and beyond," APL Photonics 4(10), 100901 (2019).

44. S. You et al., "Slide-free virtual histochemistry (Part I): development via nonlinear optics," Biomed. Opt. Express 9(11), 5240-5252 (2018).

45. Y. Liu et al., "Multimodal nonlinear microscopy by shaping a fiber supercontinuum from 900 to 1160 nm," IEEE J. Sel. Top. Quantum Electron. 18(3), 1209-1214 (2012).

46. B. Xu et al., "Quantitative investigation of the multiphoton intrapulse interference phase scan method for simultaneous phase measurement and compensation of femtosecond laser pulses," J. Opt. Soc. Am. B 23(4), 750-759 (2006).

47. X. Shi et al., "Temporal femtosecond pulse shaping dependence of laser-induced periodic surface structures in fused silica," J. Appl. Phys. 116(3), 033104 (2014).

48. A. Weiner, "Ultrafast optical pulse shaping: a tutorial review," Opt. Commun. 284(15), 3669-3692 (2011).

49. V. Lozovoy, I. Pastirk, and M. Dantus, "Multiphoton intrapulse interference. IV. Ultrashort laser pulse spectral phase characterization and compensation," Opt. Lett. 29(7), 775-777 (2004).

50. H. Dana et al., "Thy1-GCaMP6 transgenic mice for neuronal population imaging in vivo," PLoS One 9(9), e108697 (2014).

51. O. Yizhar et al., "Neocortical excitation/inhibition balance in information processing and social dysfunction," Nature 477(7363), 171-178 (2011).

52. J. Rickgauer and D. Tank, "Two-photon excitation of channelrhodopsin-2 at saturation," Proc. Natl. Acad. Sci. U. S. A. 106(35), 15025-15030 (2009). 
53. A. Packer et al., "Two-photon optogenetics of dendritic spines and neural circuits," Nat. Methods 9(12), 1202-1205 (2012).

54. A. Krull, T. Buchholz, and F. Jug, "Noise2Void-learning denoising from single noisy images," in IEEE/CVF Conf. Comput. Vision and Pattern Recognit., pp. 2124-2132 (2019).

55. C. Renteria et al., "Dynamic tracking algorithm for time-varying neuronal network connectivity using wide-field optical image video sequences," Sci. Rep. 10(1), 2540 (2020).

56. B. Andrasfalvy et al., "Two-photon single-cell optogenetic control of neuronal activity by sculpted light," Proc. Natl. Acad. Sci. U. S. A. 107(26), 11981-11986 (2010).

57. J. Rickgauer, K. Deisseroth, and D. Tank, "Simultaneous cellular-resolution optical perturbation and imaging of place cell firing fields," Nat. Neurosci. 17(12), 1816-1824 (2014).

58. E. Papagiakoumou et al., "Scanless two-photon excitation of channelrhodopsin-2," Nat. Methods 7(10), 848-854 (2010).

59. E. Stuntz et al., "Endogenous two-photon excited fluorescence imaging characterizes neuron and astrocyte metabolic responses to manganese toxicity," Sci. Rep. 7(1), 1041 (2017).

60. C. Gómez et al., "Phasor analysis of NADH FLIM identifies pharmacological disruptions to mitochondrial metabolic processes in the rodent cerebral cortex," PLoS One 13(3), e0194578 (2018).

61. A. J. Bower et al., "Tracking metabolic dynamics of apoptosis with high-speed two-photon fluroescence lifetime imaging microscopy," Biomed. Opt. Express 10(12), 6408-6421 (2019).

62. A. J. Bower et al., "High-speed imaging of transient metabolic dynamics using two-photon fluorescence lifetime imaging microscopy," Optica 5(10), 1290-1296 (2018).

Yuan-Zhi Liu received his BS degree in physics in 2007 and his $\mathrm{PhD}$ in optics in 2012, both from Sun Yat-sen University, China. He was a postdoctoral researcher at Ohio State University (2012) and became a postdoctoral research associate/research scientist at University of Illinois at Urbana-Champaign from 2013 to 2019. Currently, he is works as a senior scientist at Carl Zeiss Meditec, Inc. His research interests include biomedical imaging, computational imaging, wavefront shaping, and holography 3D imaging.

Carlos Renteria received his BSE degree in biomedical engineering from Arizona State University in 2016 and his MS degree in bioengineering from the University of Illinois at Urbana-Champaign in 2019. Currently, he is a PhD candidate in the Department of Bioengineering at the University of Illinois at Urbana-Champaign, and his research interests lie in the development and use of optical imaging technologies and computational tools for imaging and perturbing neural systems.

Connor D. Courtney is a $\mathrm{PhD}$ candidate in the neuroscience program at the University of Illinois at Urbana-Champaign. In Catherine Christian-Hinman's lab, his research involves using novel optogenetic constructs to study astrocyte-neuron interactions. He received his BS degree in psychology from Indiana University in 2014.

Baher Ibrahim received his BS and MS degrees in pharmacy and pharmacognosy from Mansoura University in 2003 and 2007. In 2014, he received his PhD in pharmacology from the University of Louisiana at Monroe. He has postdoctoral training from the University of South Carolina, the University of Illinois at Urbana-Champaign, and is currently a postdoctoral fellow in the Interdisciplinary Environmental Toxicology Program (IETP) at Illinois.

Sixian You received her MSc and $\mathrm{PhD}$ degrees from University of Illinois at UrbanaChampaign, advised by Prof. Stephen Boppart and Prof. Saurabh Sinha. She is currently a postdoctoral researcher with the Computational Imaging Lab, University of California, Berkeley, sponsored by Prof. Laura Waller. Her research is focused on the development of optical imaging techniques for biomedicine.

Eric J. Chaney received his BS degree in biology from the University of Evansville in 1992. He is currently employed as a research scientist in molecular biology at the Biophotonics Imaging 
Laboratory, Beckman Institute for Advanced Science and Technology at the University of Illinois at Urbana-Champaign.

Ronit Barkalifa received her BS, MS, and PhD degrees from Ben-Gurion University of the Negev at Israel. She is a certified Clinical Research Associate (CRA), and had previous appointments as a Clinical Research Coordinator (CRC) at the Barzilai Medical Center in Israel, and a Study Manager and Research Scientist at the Biophotonics Imaging laboratory at the University of Illinois at Urbana-Champaign. She has extensive biomedical research experience, and he is currently a clinical scientist at Bristol-Myers Squibb.

Rishyashring R. Iyer is currently a $\mathrm{PhD}$ candidate at the University of Illinois at UrbanaChampaign interested in developing novel optical microscopy systems for label-free imaging of neural activity. He received his BTech in electrical and electronics engineering from National Institute of Technology Karnataka, India in 2016 and MEng in biomedical engineering from Cornell University in 2017.

Mantas Zurauskas is a research scientist in the University of Illinois at Urbana-Champaign. He received his BS degree in physics from Vilnius University in 2009, MSc degree in optics and photonics in 2011 from Imperial College, London, and $\mathrm{PhD}$ in physics from University of Kent, Canterbury. His research interests include the development of nonlinear microscopy imaging systems and applications of data science for characterizing biological samples using multidimensional data captured with optical microscopy systems.

Haohua Tu received his BS degree in engineering from Tsinghua University and his $\mathrm{PhD}$ from University of Kentucky. He is currently a principal research scientist with the Beckman Institute, University of Illinois at Urbana-Champaign. He has worked in a wide variety of disciplines during his scientific career, including optics, spectroscopy, biomedical imaging, and photonic devices, with specific strengths in optoelectronics, laser engineering, and general optical science.

Daniel A. Llano is an associate professor of Molecular and Integrative Physiology and Neuroscience at the University of Illinois at Urbana-Champaign and the Beckman Institute for Advanced Science and Technology. His laboratory investigates auditory top-down modulation in animal models using a variety of optical and electrophysiological techniques.

Catherine A. Christian-Hinman is an assistant professor of Molecular and Integrative Physiology at the University of Illinois at Urbana-Champaign. She received her $\mathrm{PhD}$ in neuroscience from the University of Virginia and completed postdoctoral training in Neurology and Neurological Sciences at Stanford University School of Medicine. Her research focuses on using cellular and circuit electrophysiological approaches to understand interactions between neural and endocrine systems, with a particular emphasis on epilepsy.

Stephen A. Boppart received his BS and MS degrees in electrical engineering from the University of Illinois at Urbana-Champaign in 1990 and 1991, followed by his $\mathrm{PhD}$ and MD from MIT and Harvard Medical School in 1998 and 2000, respectively. He is currently executive associate dean and chief diversity officer for the Carle Illinois College of Medicine, and holds the Grainger Distinguished Chair in Engineering as professor in the Departments of Electrical and Computer Engineering and Bioengineering. 